Supporting Information

\title{
Fully Optical Tunable and Flexible Composite Films for Enhanced Terahertz Control and Multifunctional Terahertz Devices
}

Weien Lail, \#*, Run Shi ${ }^{2,3, \#}$, Hao Yuan ${ }^{1}$, Gen Liu ${ }^{1}$, Abbas Amini, ${ }^{4}$ Chun Cheng ${ }^{2,3, *}$

${ }^{1}$ National Engineering Laboratory of Special Display Technology, National Key Laboratory of Advanced

Display Technology, Anhui Province Key Laboratory of Measuring Theory and Precision Instrument,

Academy of Opto-Electronic Technology, HeFei University of Technology, HeFei, 230009, People's

Republic of China

${ }^{2}$ Department of Materials Science and Engineering, Southern University of Science and Technology,

Shenzhen 518055, People's Republic of China

${ }^{3}$ Guangdong-Hong Kong-Macao Joint Laboratory for Photonic-Thermal-Electrical Energy Materials and

Devices, Southern University of Science and Technology, Shenzhen 518055, People's Republic of China

${ }^{4}$ Center for Infrastructure Engineering, Western Sydney University, Kingswood, New South Wales 2751,

Australia

*E-mail: wnlai@ @fut.edu.cn; chengc@ sustech.edu.cn

\#W.L. and R. S. contributed equally to this work 

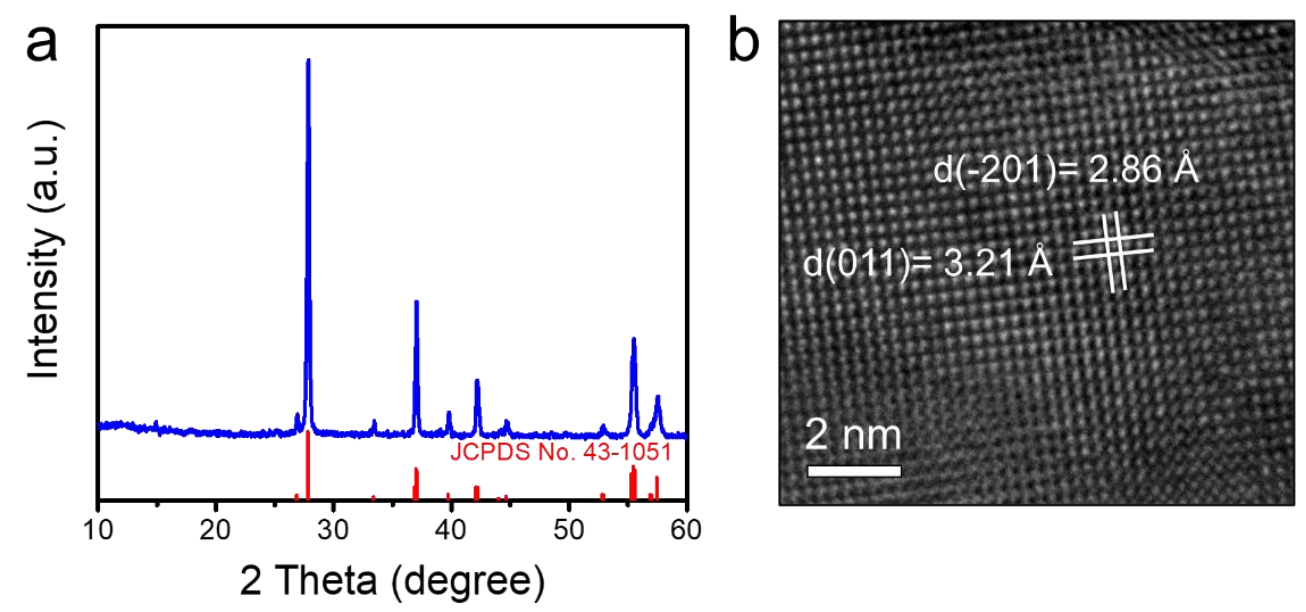

Figure S1. Characterization of the crystal structure of $\mathrm{VO}_{2}$ NWs. (a) Powder XRD pattern of as-grown $\mathrm{VO}_{2}$ NWs. (b) High-resolution transmission electron microscopy (HRTEM) image of a single $\mathrm{VO}_{2} \mathrm{NW}$ prepared by hydrothermal reactions.

As shown in Figure S1a, the X-ray diffraction peaks of as-grown $\mathrm{VO}_{2} \mathrm{NWs}$ are in good agreement with the standard diffraction lines of $\mathrm{VO}_{2}$ (M1) lattice (JCPDS No. 43-1051). Three

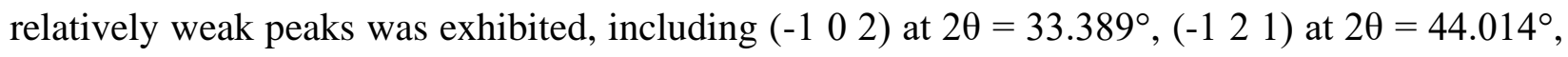
and $\left(\begin{array}{lll}0 & 2 & 1\end{array}\right)$ at $2 \theta=44.645^{\circ}$. The lattice information of single NW is characterized by HRTEM in Figure S1b, showing the good single crystallinity of the $\mathrm{VO}_{2}$ (M1) NW. 
a)

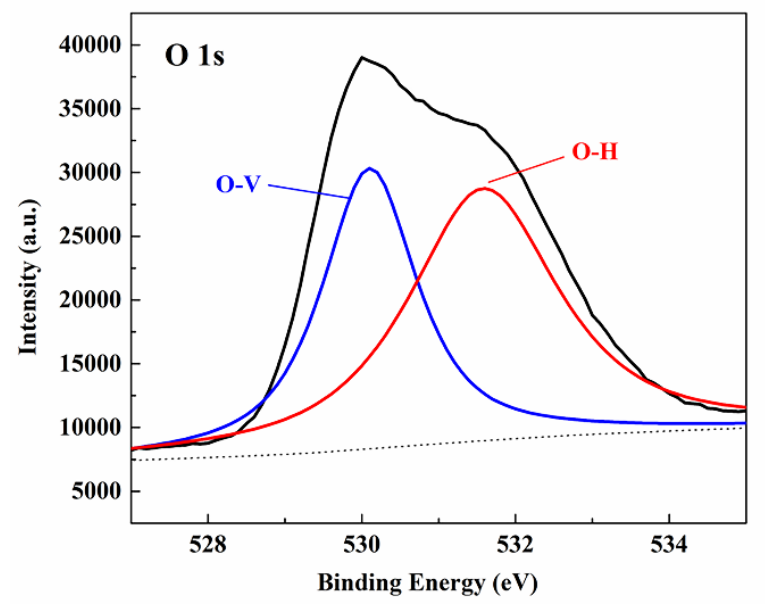

b)

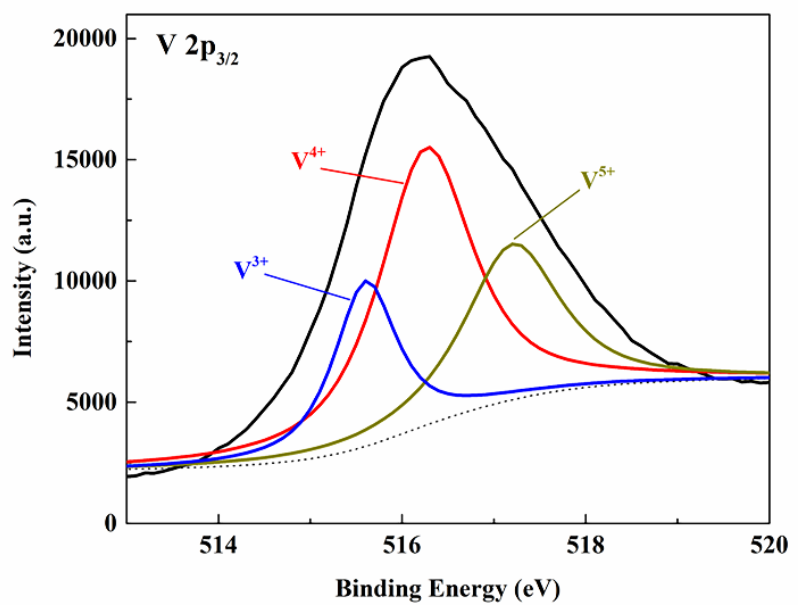

Figure S2. The fitting curves for high resolution XPS of as-grown $\mathrm{VO}_{2}$ nanowires in the region of (a) O1s, (b) V2p3/2.

As shown in Figure S2, the XPS peak of O 1s at $\sim 530 \mathrm{eV}$ can be deconvolved into two new peaks centered at $530.1 \mathrm{eV}$ and $531.6 \mathrm{eV}$, corresponding to $\mathrm{O}-\mathrm{V}$ and $\mathrm{O}-\mathrm{H}$ species ${ }^{1}$, respectively. This fact confirms the hydrogen-doping in the as-grown $\mathrm{VO}_{2}$ nanowires. In addition, $\mathrm{V}^{3+}, \mathrm{V}^{4+}$, and $\mathrm{V}^{5+}$ ions contribute to the $\mathrm{V} 2 \mathrm{p}_{3 / 2}$ peak at $\sim 516 \mathrm{eV}$. The existence of $\mathrm{V}^{3+}$ should come from the hydrogenation as reported by previous publications ${ }^{1-2}$. While the additional peak of $\mathrm{V}^{5+}$ ions should be attributed to the surface oxidation of as-grown $\mathrm{VO}_{2}$ nanowires, which is also a common phenomenon in the study of $\mathrm{VO}_{2}$ nanostructures ${ }^{3}$.

\section{Numerical Methods}

The simulation of proposed model was performed through electromagnetic calculation. In $\mathrm{THz}$ frequency range, the complex dielectric function of PVP material was obtained from a reference in literature ${ }^{4}$. For $\mathrm{VO}_{2} \mathrm{NWs}$, the relation between the effective mass $m_{\text {eff }}^{*}$ of charge carriers and $\tau$ were defined as $\mu=q_{e} \tau / m_{e f f}^{*}$, where $\mu$ was the charge mobility. According to 
the references ${ }^{5-6}$, the high-frequency dielectric constant could be equal to the dielectric function of the insulating domain, $\varepsilon_{\infty}=\varepsilon_{i}=9.0$. Then, we used the parameters of $\mathrm{VO}_{2}$ as $m_{e f f}^{*}=2 m_{e}$, $\mu \approx 2 \mathrm{~cm}^{2} /(V \cdot s)$ and $N \approx 2.0 \times 10^{21} \mathrm{~cm}^{-3}$ to calculate the dielectric function $\varepsilon_{m}$ of $\mathrm{VO}_{2} \mathrm{NWs}$ in the metallic state in term of the theoretical model in the paper. Finally, we used the above obtained parameters to fit the experimental results with the electromagnetic calculation. Herein, the proposed NIR light driven composite film was mainly applied for the common $\mathrm{THz}$ communication frequency range of 0.32-0.5 THz. The spectral resolution and accuracy obtained by the $\mathrm{CW} \mathrm{THz}$ system are better than the terahertz time-domain spectroscopy, so the paper only showed the experimental results from the $\mathrm{CW}$ THz system.

The transmission stability $P_{s}$ of the device is expressed as $P_{s}=\left(\frac{1}{m} \sum_{\omega=\omega_{1}}^{\omega_{m}} T_{N}(\omega) / T_{0}(\omega)\right) \cdot 100 \%$, where $T_{N}$ is the transmittance of the device at bending period $\mathrm{N}$ under light irradiation, $T_{0}$ is the transmittance of the device in the original condition before bending began under light irradiation, $\omega$ is the angular frequency of incident $\mathrm{THz}$ waves, and $\mathrm{m}$ is the number of frequency sampling points. According to the experimental results, the stability of the composite film is good during an IR excitation across these bending attempts.

\section{References}

(1) Peic, A.; Staff, D.; Risbridger, T.; Menges, B.; Peter, L. M.; Walker, A. B.; Cameron, P. J. Real-time optical waveguide measurements of dye adsorption into nanocrystalline $\mathrm{TiO} 2$ films with relevance to dye-sensitized solar cells. J. Phys. Chem. C 2011, 115 (3), 613-619.

(2) Li, Z.; Guo, Y.; Hu, Z.; Su, J.; Zhao, J.; Wu, J.; Wu, J.; Zhao, Y.; Wu, C.; Xie, Y. Hydrogen treatment for superparamagnetic VO2 nanowires with large room - temperature magnetoresistance. Angew. Chem. Int. Ed. 2016, 55 (28), 8018-8022. 
(3) Fan, S.; Fan, L.; Li, Q.; Liu, J.; Ye, B. The identification of defect structures for oxygen pressure dependent VO2 crystal films. Appl. Surf. Sci. 2014, 321, 464-468.

(4) Chen, X.-L.; Chen, X.; Zhu, Z.-Y. THz-TDS spectra study of polymer materials with different polarity. J. Infrared Millimeter Waves 2013, 32, 150-153.

(5) Jepsen, P. U.; Fischer, B. M.; Thoman, A.; Helm, H.; Suh, J.; Lopez, R.; Haglund Jr, R. Metal-insulator phase transition in a VO2 thin film observed with terahertz spectroscopy. Phys. Rev. B 2006, 74 (20), 205103.

(6) Lourembam, J.; Srivastava, A.; Rotella, H.; Venkatesan, T.; Chia, E. E. New insights into the diverse electronic phases of a novel vanadium dioxide polymorph: a terahertz spectroscopy study. Sci. Rep-UK 2015, 5, 9182. 